\title{
The Distribution of IOL Center and Its Relationship with Internal Ocular Objective Visual Quality in Cataract Patients
}

\author{
Tan Long \\ Xin Gu \\ the First Affiliated Hospital of Northwest University \\ Wei Wei \\ the First Affiliated Hospital of Northwest University

\section{Ting Ma} \\ the First Affiliated Hospital of Northwest University \\ Rui Wang ( $\square$ wangmeiyi1986@outlook.com) \\ the First Affiliated Hospital of Northwest University
}

the First Affiliated Hospital of Northwest University https://orcid.org/0000-0003-0062-7463

\section{Research article}

Keywords: intraocular lens, visual quality, cataract

Posted Date: February 16th, 2021

DOI: https://doi.org/10.21203/rs.3.rs-236882/v1

License: (c) (i) This work is licensed under a Creative Commons Attribution 4.0 International License. Read Full License 


\section{Abstract}

Background: To investigate the distribution of the center of the intraocular lens (IOL) after phacoemulsification, and to assess the correlation between the center of IOL and preoperative angle kappa, angle alpha, and objective internal visual quality, respectively, in cataract patients with monofocal and bifocal IOLs implantation.

Methods: Prospective cross-section cases series. One hundred and thirty-seven eyes of 107 patients who underwent phacoemulsification were included. Preoperative angle kappa and alpha, postoperative internal ocular aberrations, internal objective visual quality, and the center of IOL relative to the visual axis (CIV) was evaluated using iTrace system. Independent sample $t$-tests and Pearson correlations were performed.

Results: Locations of CIV were scattered in all directions centered on corneal light reflection for both C-Loop designed IOL and plate-haptic designed IOL. No correlations were found between CIV and preoperative angle kappa and alpha in both magnitude and orientation. No correlations were found between CIV and postoperative internal ocular aberrations (astigmatism, coma, and trefoil). In the bifocal IOLs group, the CIV was negatively correlated to the internal Strehl ratio at $3 \mathrm{~mm}$; however, it was not correlated to the Strehl ratio at $5 \mathrm{~mm}$. The magnitude of CIV was positively correlated to the length of the optic axis.

Conclusions: CIV was not predictable according to angle kappa and alpha before cataract surgery. CIV was not related to internal ocular aberration, but large CIV may lead to light scattering due to steps between diffractive rings in patients with small pupil sizes. The magnitude of CIV may be greater in cataract patients with longer optic axis.

Trial registration: retrospectively registered.

\section{Background}

Premium intraocular lens (IOL) decentration, which can result in significant visual disturbances, is one of the main reasons of patient dissatisfaction. A well-centered IOL is crucial for good visual quality because the light path (the line between the fixation target and the fovea) has to pass thought the center of the multifocal IOL ( $\mathrm{mlOL}$ ). Otherwise, patients may experience unpleasant photic phenomena. ${ }^{1,2}$ These phenomena are common causes of dissatisfaction in patients with mIOLs implantation, even when uncorrected visual acuity is excellent. ${ }^{3}$ In patients with a large angle kappa, functional IOL decentration can happen if the fovea-centric ray does not pass though IOL center, ${ }^{1}$ and induces aberrations, glare and halo. ${ }^{4-6}$ However, it was also found that many patients with a large angle kappa were asymptomatic. ${ }^{1}$ Angle alpha, which is defined as the intersection of the visual axis with the optic axis, is believed to represent the center of the capsular bag. A recent study showed that the location of IOL was correlated to angle alpha. ${ }^{7}$ It has been proposed that angle kappa and alpha should be considered during preoperative assessment for mIOLs implantation to better predict outcomes. ${ }^{8-10}$

Are there any potential factors that impact the center of IOL in patients who were considered good candidates for premium IOL implantation after passing strict and adequate preoperative assessment? Is IOL center predictable during preoperative assessment in cataract patients? Does a well-centered mIOLs, which has been confirmed during surgery and follow-up visit, lead to good visual outcome? We know little about how angle kappa and alpha impact the visual quality after mIOLs implantation. The aim of this study was therefore to look into the relationship among the center of IOL, angle kappa and angle alpha, and check the correlation between the center of IOL and internal ocular aberrations and objective visual quality.

\section{Methods}


In this cross-sectional cases series study, patients who had been scheduled for cataract surgery from December 2018 to December 2019 were included. All patients were subjected to phacoemulsification surgery and were divided into two groups depending on the type of IOL. There were 91 eyes of 74 patients in group 1 with the implantation of three different designed aspherical monofocal IOLs including ZCB00 (Johnson \& Johnson Vision, USA), 509MP (Carl Zeiss Meditec SAS, France) and 409MP (Carl Zeiss Meditec SAS, France). There were 46 eyes of 33 patients in group 2 that received implantation of a diffraction optical design bifocal IOLs including ZMB00 (Johnson \& Johnson Vision, USA), 809MP (Carl Zeiss Meditec SAS, France). This study was performed in accordance with the tenets of the Declaration of Helsinki and has been approved by the local ethics committee (The First Affiliated Hospital of Northwest University, Xi'an, China). Written informed consent was obtained from all patients.

Inclusion criteria were as follows: 1) patients' preoperative lens opacity grading greater than C2N2P0 according to Lens Opacity Classification System III, 2) corneal astigmatism less than 0.75 diopter, 3) no history of ocular disease other than cataract, 4) no previous ocular surgery, 5) no intraoperative and postoperative complications. Exclusion criteria included poor cooperation during examination, inability to obtain data due to severe lens opacity preoperatively, notable IOL decentration or tilt under slit lamp evaluation during the follow-up, and patients lost to follow-up.

\section{Preoperative and Postoperative Assessment}

Preoperative assessment was taken within 2 days before surgery and postoperative assessment was taken at a 3 month follow-up visit. All patients underwent a full ophthalmologic examination, including slit-lamp examination for both anterior and posterior segments of the eye, uncorrected distance and near visual acuity, best corrected distance and near visual acuity, intraocular pressure (IOP), OCT (Heidelberg engineering, Germany), and optical biometry (Lenstar LS 900, HAAGSTREIT, USA). Visual quality and ocular aberration were determined by iTrace (Tracey Technologies, Houston, TX, USA).

\section{IOL Selection}

Three models of aspherical monofocal IOLs were used in group 1. The difference between those three models of IOLs was the ability of correcting spherical aberration. The spherical aberrations of ZCB00, 509MP and 409MP are $-0.27 \mu \mathrm{m}$, $-0.18 \mu \mathrm{m}$ and $0 \mu \mathrm{m}$, respectively. Two models of bifocal IOLs were used in group 2. The spherical aberrations of ZMB00 and $809 \mathrm{MP}$ are $-0.27 \mu \mathrm{m}$ and $-0.18 \mu \mathrm{m}$, respectively. During preoperative examination, the corneal spherical aberration at $6 \mathrm{~mm}$ was measured by iTrace, and the model of IOLs was decided with the goal to remove as much of corneal spherical aberration as possible.

\section{Surgical Procedure}

The same experienced surgeon (Ting Ma) performed all the operations under topical anesthesia (Benoxil $0.4 \%$ solution, 3 times, Santen) using a standard technique of phacoemulsification. A $2.4 \mathrm{~mm}$ clear corneal incision was made with a diamond knife at the $135^{\circ}$ axis and an auxiliary incision was made at $45^{\circ}$ axis. All IOLs were implanted into the capsule bag, and the whole circumference of the IOL optical zone was covered by the anterior capsule. Antibiotic (Cravit 0.5\%, Santen), NASIDs (Pranopulin, Senju), and artificial tears (Hycosan, $0.1 \%$, Eusan) were used for 4 weeks by all patients postoperatively. All operations were successful and there were no intraoperative complications.

\section{Evaluation indicators}

Angle alpha, angle kappa, internal ocular aberrations, internal modulation transfer function (MTF) and Strehl ratio of all participants were measured using iTrace aberrometer by an experienced technician (Xin Gu) before and after the surgery. Angle kappa and alpha were measured under scotopic condition. After that, the patient's pupil was dilated to more than $6.5 \mathrm{~mm}$ using tropicamide (Mydrin-P, Santen) to obtain internal ocular aberrations, internal MTF and Strehl ratio for both $3 \mathrm{~mm}$ and $5 \mathrm{~mm}$ scanning zones. The iTrace system uses optical ray-tracing technology and measures the point spread function (PSF) of the eye with its retinal spot detection. Three measurements of each eye were captured, and the best scan (the image with the best quality peaks for individual points) was chosen for the final analysis. The center of the cornea 
and pupil relative to corneal light reflection were presented in magnitude and orientation. Root-mean-square (RMS) terms of total higher-order aberrations (HOAs) of internal eye and individual aberrations (astigmatism, coma, and trefoil) of internal eye were evaluated. The MTF curves due to higher order aberrations and Strehl ratio were evaluated for the internal eye.

\section{Center of IOL}

The distance and orientation of the center of IOL relative to the visual axis (CIV), was determined using iTrace by the same technician (Xin $\mathrm{Gu}$ ) at the time of the last follow-up (3 months). The patient's pupil was dilated to more than $6.5 \mathrm{~mm}$ so the entire optical zone of the IOL could be observed. A radial ruler was used to show the edge of the optical zone, and the center of the IOL was determined at the same time. The distance and orientation relative to the visual axis were calculated. See Fig. 1 for details.

\section{Statistical Analysis}

The mean and standard deviation of angle kappa, angle alpha, CIV, aberrations, MTF and Strehl ratio were calculated. Mean values were used for population analysis. Student paired $t$ tests were performed to test for significant differences in the visual acuity, IOP pre- and postoperatively. Independent sample $t$ tests were performed to test for significant differences in the magnitude and the orientation of CIV, angle kappa and angle alpha. Pearson correlations were performed for correlation analysis. A $P$ value less than 0.05 was regarded as statistically significant. All statistics were calculated using SPSS 17.0. The datasets used for analysis during the current study are available from the corresponding author on reasonable request.

\section{Results}

One hundred and forty-seven eyes from 113 patients underwent phacoemulsification, 4 patients did not return for the follow-up examination after surgery, and 2 patients did not cooperate (poor fixation) during examination. At the end of this study, data of 137 eyes (77 right eyes and 60 left eyes) from 107 patients were included. Ninety-one eyes of 74 patients ( 30 male and 44 female) were implanted with monofocal IOLs, and 46 eyes ( 24 right eyes and 22 left eyes) of 33 patients (13 male and 20 female) were implanted with bifocal IOLs. The mean age was $67.38 \pm 12.16$ (range 20 to 86 years) in the monofocal group and $66.06 \pm 9.55$ (range 37 to 83 years) in the bifocal group. In the monofocal group, 43 eyes were implanted with one-piece plate-haptic lenses and 48 eyes were implanted with one-piece C-Loop haptic lenses. In the bifocal group, 36 eyes were implanted with one-piece plate-haptic lenses and 10 eyes were implanted with one-piece CLoop haptic lenses. In the monofocal group, preoperative uncorrected distance visual acuity (UDVA) was significantly lower than postoperative UDVA ( $0.84 \pm 0.39$ versus $0.17 \pm 0.11$ Mean $\pm \mathrm{SD}, \log \mathrm{MAR}, t=12.890, P=0.000)$, and preoperative corrected distance visual acuity (CDVA) was significantly lower than postoperative CDVA $(0.54 \pm 0.33$ versus $0.07 \pm 0.09$, Mean $\pm S D, \log M A R, t=14.383, P=0.000)$. In the bifocal group, preoperative UDVA was $0.75 \pm 0.48$, which was significantly lower than postoperative UDVA $(0.15 \pm 0.19$, Mean $\pm \mathrm{SD}$, logMAR, $t=6.460, P=0.000)$, and preoperative CDVA $₫ 0.44 \pm$ 0.39 , Mean \pm SD, logMAR was significantly lower than postoperative CDVA $(0.10 \pm 0.24$, Mean \pm SD, $\log M A R, t=3.426, P=$ 0.002 ). Postoperative uncorrected near visual acuity (UNVA) was $0.20 \pm 0.20$ (Mean $\pm S D$, logMAR) in the bifocal group. Table 1 shows the ocular bio-metric data. 
Table 1

Ocular Biometric Data of All Patients

\begin{tabular}{|lllll|}
\hline \multirow{2}{*}{$\begin{array}{l}\text { Parameter } \\
(\mathrm{mm})\end{array}$} & \multicolumn{2}{l}{ Monofocal IOL $(\mathbf{n}=\mathbf{9 1})$} & \multicolumn{2}{l|}{ Bifocal IOL(n= 46) } \\
\cline { 2 - 5 } & Mean \pm SD & Range & Mean \pm SD & Range \\
\hline AL & $23.77 \pm 1.78$ & $21.12,30.67$ & $23.92 \pm 1.80$ & $21.06,28.17$ \\
\hline WTW & $11.43 \pm 0.51$ & $9.77,12.61$ & $11.55 \pm 0.60$ & $9.76,12.98$ \\
\hline ACD & $3.04 \pm 0.40$ & $2.13,4.14$ & $3.21 \pm 0.53$ & $2.14,4.41$ \\
\hline LT & $4.39 \pm 0.48$ & $3.40,5.29$ & $4.31 \pm 0.52$ & $3.15,5.18$ \\
\hline Kappa* & $0.30 \pm 0.16$ & $0.03,0.51$ & $0.25 \pm 0.20$ & $0.02,0.98$ \\
\hline Alpha* & $0.35 \pm 0.14$ & $0.01,0.66$ & $0.29 \pm 0.14$ & $0.01,0.73$ \\
\hline AL: Axial length; WTW: White to white; ACD: Anterior chamber depth; LT: Lens thickness; \\
\hline *: Only the magnitude of angle kappa and alpha is shown in the table \\
\hline
\end{tabular}

In group 1, magnitudes of preoperative angles kappa and alpha were significantly different from the magnitude of CIV; and no correlations were found between CIV and the magnitudes of preoperative angles kappa and alpha. There were significant differences between the orientation of CIV and orientations of preoperative angles kappa and alpha; and no correlations were found (Table 2). The distributions of CIV, angle kappa and angle alpha of all 91 eyes of Group 1 were graphed in polar coordinate diagrams in Fig. 2. Locations of angle kappa and alpha were distributed mostly on the temporal side of the corneal light reflection, while angle alpha clustered along the horizontal line. The location of IOL centers showed a broader distribution. For Group 1, the distribution of CIV of two different designed IOL is shown in Fig. 3. No significant differences were found between plate-haptic IOL and C-Loop haptic IOL for CIV in both magnitude and orientation (Table 3). There were no significant differences between males and females or right eyes and left eyes for CIV in magnitude and orientation (Table 3). Age did not correlate with CIV magnitude and orientation $(r=-0.005, P=$ $0.096, r=-0.006, P=0.952$, respectively). The length of the optic axis positively correlated with CIV in magnitude ( $r=$ $0.236, P=0.028)$, while no correlation was found between the length of the optic axis and CIV in orientation $(r=-0.139, P=$ $0.200)$.

Table 2

Correlations between CIV and Angle Kappa, Angle Alpha

\begin{tabular}{|c|c|c|c|c|c|c|c|}
\hline & & & CIV & $t$ & $P$ & $r$ & $P$ \\
\hline \multirow{2}{*}{$\begin{array}{l}\text { Magnitude } \\
(\mathrm{mm})\end{array}$} & $\mathbf{K}$ & $0.30 \pm 0.16$ & \multirow[t]{2}{*}{$0.22 \pm 0.13$} & -3.764 & 0.000 & 0.080 & 0.449 \\
\hline & $\mathbf{a}$ & $0.35 \pm 0.14$ & & -6.359 & 0.000 & -0.043 & 0.683 \\
\hline \multirow{2}{*}{$\begin{array}{l}\text { Orientation } \\
\text { (degree) }\end{array}$} & K & $208.15 \pm 94.74$ & \multirow[t]{2}{*}{$168.31 \pm 106.94$} & -2.951 & 0.004 & 0.188 & 0.074 \\
\hline & $a$ & $205.60 \pm 108.81$ & & -2.400 & 0.018 & 0.056 & 0.600 \\
\hline
\end{tabular}


Table 3

CIV in Different Design, Gender, and Eye

\begin{tabular}{|c|c|c|c|c|c|c|c|}
\hline & & magnitude of CIV & $t$ & $P$ & orientation of CIV & $t$ & $P$ \\
\hline \multirow[t]{2}{*}{ Design of IOL } & Plate-haptic & $0.20 \pm 0.12$ & \multirow[t]{2}{*}{-1.265} & \multirow[t]{2}{*}{0.209} & $151.56 \pm 104.01$ & \multirow[t]{2}{*}{-1.422} & \multirow[t]{2}{*}{0.158} \\
\hline & C-Loop haptic & $0.24 \pm 0.13$ & & & $183.31 \pm 108.38$ & & \\
\hline \multirow[t]{2}{*}{ Gender } & male & $0.21 \pm 0.12$ & \multirow[t]{2}{*}{-0.881} & \multirow[t]{2}{*}{0.381} & $180.44 \pm 108.86$ & \multirow[t]{2}{*}{0.875} & \multirow[t]{2}{*}{0.384} \\
\hline & female & $0.23 \pm 0.13$ & & & $160.36 \pm 105.91$ & & \\
\hline \multirow[t]{2}{*}{ Eye } & right & $0.22 \pm 0.11$ & \multirow[t]{2}{*}{0.102} & \multirow[t]{2}{*}{0.919} & $163.68 \pm 91.92$ & \multirow[t]{2}{*}{-0.486} & \multirow[t]{2}{*}{0.628} \\
\hline & left & $0.22 \pm 0.16$ & & & $174.76 \pm 125.97$ & & \\
\hline
\end{tabular}

The correlation between CIV and internal individual ocular aberrations, internal total HOA, internal MTF due to HOA, and internal Strehl ratio were determined. In Group 1, there was no correlation between the magnitude of CIV and astigmatism $(r=0.133, P=0.282)$, coma $(r=0.091, P=0.465)$, trefoil $(r=0.039, P=0.751)$, HOA $(r=0.072, P=0.565)$, MTF due to HOA $(r=-0.174, P=0.159)$ and Strehl ratio $(r=-0.113, P=0.364)$ at $3 \mathrm{~mm}$, respectively. No correlation was found between the magnitude of CIV and astigmatism $(r=0.123, P=0.366)$, coma $(r=0.202, P=0.135)$, trefoil $(r=0.034, P=0.806), \mathrm{HOA}(r=$ $0.098, P=0.474)$, MTF due to HOA $(r=-0.195, P=0.149)$ and Strehl ratio $(r=-0.212, P=0.117)$ at $5 \mathrm{~mm}$, respectively. In group 2 , there was no correlation between the magnitude of CIV and astigmatism $(r=0.062, P=0.754)$, coma $(r=0.205, P=$ 0.277), trefoil $(r=0.257, P=0.171), \mathrm{HOA}(r=0.333, P=0.072)$, and MTF due to HOA $(r=-0.198, P=0.294)$ at $3 \mathrm{~mm}$, respectively. The Strehl ratio of internal eye at $3 \mathrm{~mm}$ was negatively correlated to the magnitude of CIV $(r=-0.368, P=$ 0.045). No correlation was found between the magnitude of CIV and all variables at $5 \mathrm{~mm}$ in patient with bifocal IOLs (Table 4). . 
Table 4

Correlations between CIV and Aberrations and Objective Visual Quality

\begin{tabular}{|c|c|c|c|c|c|c|c|c|c|}
\hline & & \multicolumn{4}{|l|}{ Group 1} & \multicolumn{4}{|l|}{ Group 2} \\
\hline & & $\begin{array}{l}\text { Parameters } \\
\text { of internal } \\
\text { eye }(n=91)\end{array}$ & $\begin{array}{l}\text { Magnitude } \\
\text { of CIV }\end{array}$ & $r$ & $\mathrm{p}$ & $\begin{array}{l}\text { Parameters } \\
\text { of internal } \\
\text { eye }(n=46)\end{array}$ & $\begin{array}{l}\text { Magnitude } \\
\text { of CIV }\end{array}$ & $r$ & $\mathrm{p}$ \\
\hline \multirow[t]{6}{*}{3 mm } & $\mathrm{HOA}$ & $0.16 \pm 0.11$ & \multirow{12}{*}{$\begin{array}{l}0.21 \pm \\
0.13\end{array}$} & 0.072 & 0.565 & $0.11 \pm 0.07$ & \multirow{12}{*}{$\begin{array}{l}0.24 \pm \\
0.11\end{array}$} & 0.333 & 0.072 \\
\hline & Astigmatism & $0.21 \pm 0.14$ & & 0.133 & 0.282 & $0.14 \pm 0.08$ & & 0.062 & 0.754 \\
\hline & Coma & $0.09 \pm 0.08$ & & 0.091 & 0.465 & $0.06 \pm 0.03$ & & 0.205 & 0.277 \\
\hline & Trefoil & $0.09 \pm 0.06$ & & 0.039 & 0.751 & $0.06 \pm 0.05$ & & 0.257 & 0.171 \\
\hline & MTF* & $0.35 \pm 0.13$ & & -0.174 & 0.159 & $0.43 \pm 0.12$ & & -0.198 & 0.294 \\
\hline & Strehl ratio & $0.12 \pm 0.10$ & & -0.113 & 0.364 & $0.22 \pm 0.17$ & & -0.368 & $0.045^{\#}$ \\
\hline \multirow[t]{6}{*}{$5 \mathrm{~mm}$} & $\mathrm{HOA}$ & $0.44 \pm 0.32$ & & 0.098 & 0.474 & $0.30 \pm 0.11$ & & 0.191 & 0.395 \\
\hline & Astigmatism & $0.52 \pm 0.34$ & & 0.123 & 0.366 & $0.32 \pm 0.15$ & & 0.191 & 0.394 \\
\hline & Coma & $0.22 \pm 0.17$ & & 0.202 & 0.135 & $0.13 \pm 0.07$ & & 0.381 & 0.080 \\
\hline & Trefoil & $0.21 \pm 0.16$ & & 0.034 & 0.806 & $0.16 \pm 0.10$ & & 0.181 & 0.419 \\
\hline & MTF* & $0.27 \pm 0.11$ & & -0.195 & 0.149 & $0.31 \pm 0.07$ & & -0.300 & 0.175 \\
\hline & Strehl ratio & $0.04 \pm 0.03$ & & -0.212 & 0.117 & $0.05 \pm 0.02$ & & -0.336 & 0.126 \\
\hline \multicolumn{10}{|c|}{ HOA: higher-order aberrations; MTF: modulation transfer function; } \\
\hline \multicolumn{10}{|c|}{ *: MTF due to higher-order aberrations } \\
\hline \multicolumn{10}{|c|}{$\#: P<0.05$} \\
\hline
\end{tabular}

\section{Discussion}

Angle alpha is defined as the angular distance between optic and visual axes and was first measured by Tscherning using an ophthalmophakometer. ${ }^{11}$ It can indicate the distance between the center of the capsular and visual axis because theoretically the optic axis passes through the center of curvature of each surface - the anterior and posterior corneal surfaces, as well as anterior and posterior crystalline lens surfaces in the system of the eye. A recent study showed that the location of IOL is correlated to angle alpha. ${ }^{7}$ We investigated the correlation between the CIV and preoperative angle alpha for both magnitude and orientation, but no correlations were found. CIV showed a diffuse distribution (Fig. 2). Plotting the center of IOL relative to the corneal light reflection showed wide scattering without a discernible pattern (Fig. 2G-I), whereas the values for angle alpha fell on a narrow horizontal line on the temporal sides of corneal light reflection (Fig. 2D-F). This finding suggests that decentration of IOL relative to visual axis may not be related to a larger angle alpha. Further studies need to be done for the relationship between large angle alpha and visual quality.

Angle kappa is the intersection of the visual axis and the pupillary axis. ${ }^{12}$ It can be measured by the distance from the pupil center to a point that is very close to the visual axis, such as the corneal vertex or corneal light reflection. ${ }^{12}$ In previous studies, it has been found that poor satisfaction after multifocal lens implantation may be related to large angle kappa. ${ }^{1}$

${ }^{13}$ Qi Y et al reported that with preoperative angle kappa greater than $0.5 \mathrm{~mm}$, visual quality decreased in patients implanted with trifocal diffractive IOLs. ${ }^{5}$ The association between angle kappa and bad visual quality is unclear. Prakash $\mathrm{G}$ et al proposed the concept of functional IOL decentration, indicating that the light passes through paracentral IOL rings 
or through the edge of the IOL rings even when the IOL seems to be well-centered in the capsular bag in those eyes with greater angle kappa. ${ }^{1}$

In this study, we found no correlation between the CIV and angle kappa in magnitude and orientation. The distribution of angle kappa in both eyes was similar to CIV. However, when the left eyes and right eye were checked separately, locations of angle kappa distributed mostly to the temporal side of corneal light reflection, while the center of IOL were scattered in all directions relative to the corneal light reflection (Fig. 2). Similar distributions of CIV were found in both C-Loop designed IOLs and plate-haptic designed IOL (Fig. 3). These findings indicate that the postoperative location of an IOL maybe affected by factors such as the size of capsular bag, asymmetric zonular weakness, or uneven capsular contraction rather than angles kappa and alpha.

The PSF, which in an optical system represents the intensity distribution of light from a point-like object projected onto the retina, indicates the extent of the blurring of vision. Calculating the maximum intensity of this observed retina image and dividing it by the maximum intensity of a diffraction-limited ideal optic system yields the Strehl ratio. ${ }^{14}$ Aberrations are not the only factor contributing to the PSF, but diffraction, scatter, and phase shift are also important. In this study, the correlation analysis showed that astigmatism, coma, trefoil and HOA from internal eye were not correlated with the magnitude of CIV in both monofocal IOL group and bifocal IOL group. It should be pointed out that the magnitude of CIV was 0.02 to $0.85 \mathrm{~mm}$ and 0.06 to $0.48 \mathrm{~mm}$ in monofocal and bifocal group, respectively. This may not be large enough to induce large internal aberrations. However, the Strehl ratio of the internal eye was negatively correlated to the magnitude of $\mathrm{CIV}$ at $3 \mathrm{~mm}$ pupil size in the bifocal IOL group. This indicates that a small decentration of a bifocal IOL may affect visual quality compared to a monofocal IOL, and larger CIV may reduce visual quality by reasons other than aberrations, such as scatter or phase shift. No correlation was found between the magnitude of CIV and the Strehl ratio of internal eye at $5 \mathrm{~mm}$ in patients with bifocal IOLs. The reasons maybe that the main factors contribute to visual quality become more complicated due to a larger pupil size and the impact of CIV on visual quality is reduced. Hence, when the magnitude of $\mathrm{CIV}$ is large and the pupil size is small, even a relatively minor decentration may cause the light to pass through other diffractive rings rather than the central optical region of the $\mathrm{mIOL}$.

Neither gender nor age were significantly correlated to the magnitude and the orientation of the center of the IOL. However, the length of the optic axis positively correlated with the center of IOL in magnitude, which indicates that the location of the IOL may be more variable in patients with longer optical axis than in normal patients.

\section{Conclusion}

In summary, the center of IOL relative to the visual axis was not related to angle kappa and angle alpha. A large CIV may be a risk factor for unpleasant visual phenomena in patients with small pupil sizes. Although our study showed that in cataract patients the magnitude of angle kappa does change after the cataract surgery, ${ }^{15}$ surgeons should pay more attention to patients with small pupil sizes and long optical axis.

\section{Abbreviations}

CDVA

corrected distance visual acuity

CIV

center of IOL relative to the visual axis

$\mathrm{HOA}$

higher-order aberrations

IOL

intraocular lens 
IOP

intraocular pressure

$\mathrm{mIOL}$

multifocal IOL

MTF

modulation transfer function

PSF

point spread function

RMS

root-mean-square

UDVA

uncorrected distance visual acuity

\section{Declarations}

- Ethics approval and consent to participate

This study was performed in accordance with the tenets of the Declaration of Helsinki and has been approved by the local ethics committee (The First Affiliated Hospital of Northwest University, Xi'an, China).

- Consent for publication

The consent for publication have been obtained.

- Availability of data and materials

The datasets generated and analysed during the current study are not publicly available due to the privacy but are available from the corresponding author on reasonable request.

- Competing interests

The authors declare that they have no competing interests.

- Funding

Shaanxi Province Social Development and Technology of Research Project (2018SF-216 (WW), 2020SF-264 (RW) )

- Authors' contributions

TL have made substantial contributions to the acquisition, analysis, interpretation of data and drafted the work

XG have made substantial contributions to the iTrace test and the interpretation of the data

WW have made substantial contributions to the other test and the interpretation of the data

TM have made substantial contributions to the conception

RW have have made substantial contributions to the design of the work and substantively revised it

- Acknowledgements

The authors thank Professor Tatjana C. Jakobs for providing English-language editing. 


\section{References}

1. Prakash G, Prakash DR, Agarwal A, Kumar DA, Agarwal A, Jacob S. Predictive factor and kappa angle analysis for visual satisfactions in patients with multifocal IOL implantation. Eye (Lond). 2011;25:1187-93.

2. Liu XM, Xie LX, Huang YS. Effects of decentration and tilt at different orientations on the optical performance of a rotationally asymmetric multifocal intraocular lens. J Cataract Refract Surg. 2019;45:507-14.

3. de Vries NE, Webers CA, Touwslager WR, Bauer NJ, de Brabander J, Berendschot TT, Nuijts RM. Dissatisfaction afterimplantation of multifocal intraocular lenses. J Cataract Refract Surg. 2011;37:859-65.

4. Karhanová M, Marešová $K$, Pluháček F, Mlčák $P$, Vláčil $O$, Sín $M$. The importance of angle kappa for centration of multifocal intraocular lenses. Cesk Slov Oftalmol. 2013;69:64-8.

5. Qi Y, Lin J, Leng L, Zhao G, Wang Q, Li C, Hu L. Role of angle $\mathrm{k}$ in visual quality in patients with a trifocal diffractive intraocular lens. J Cataract Refract Surg. 2018;44:949-54.

6. Fu Y, Kou J, Chen D, Wang D, Zhao Y, Hu M, Lin X, Dai Q, Li J, Zhao YE. Influence of angle kappa and angle alpha on visual quality after implantation of multifocal intraocular lenses. J Cataract Refract Surg. 2019;45:1258-64.

7. Wang L, Guimaraes de Souza R, Weikert MP, Koch DD. Evaluation of crystalline lens and intraocular lens tilt using a swept-source optical coherence tomography biometer. J Cataract Refract Surg. 2019;45:35-40.

8. Karhanová M, Pluháček F, Mlčák P, Vláčil O, Šín M, Marešová K. The importanceof angle kappa evaluation for implantation of diffractive multifocalintra-ocular lenses using pseudophakic eye model. Acta Ophthalmol. 2015;93:e123-8.

9. Tchah H, Nam K, Yoo A. Predictive factors for photic phenomena afterrefractive, rotationally asymmetric, multifocal intraocular lens implantation. Int J Ophthalmol. 2017;10:241-5.

10. Piracha AR. Using angle alpha in premium IOL screening. Cataract Refractive Surgery Today. 2016;16:24-5.

11. Tscherning M. Physiologic Optics (trans. C. Weiland). 4th ed. Philadelphia: Keystone; 1924.

12. Hashemi H, KhabazKhoob M, Yazdani K, Mehravaran S, Jafarzadehpur E, Fotouhi A. Distribution of angle kappa measurements with Orbscan II in a population-based survey. J RefractSurg. 2010;26:966-71.

13. Prakash G, Agarwal A, Prakash DR, Kumar DA, Agarwal A, Jacob S. Role of angle kappa in patient dissatisfaction with refractive-design multifocal intraocular lenses. J Cataract Refract Surg. 2011;37:1739-40.

14. Breyer DRH, Kaymak H, Ax T, Kretz FTA, Auffarth GU, Hagen PR. Multifocal Intraocular Lenses and Extended Depth of Focus Intraocular Lenses. Asia Pac J Ophthalmol (Phila). 2017;6:339-49.

15. Wang R, Long T, Gu X, Ma T. Changes in angle kappa and angle alpha before and after cataract surgery. J Cataract Refract Surg. 2020;46:365-71.

\section{Figures}



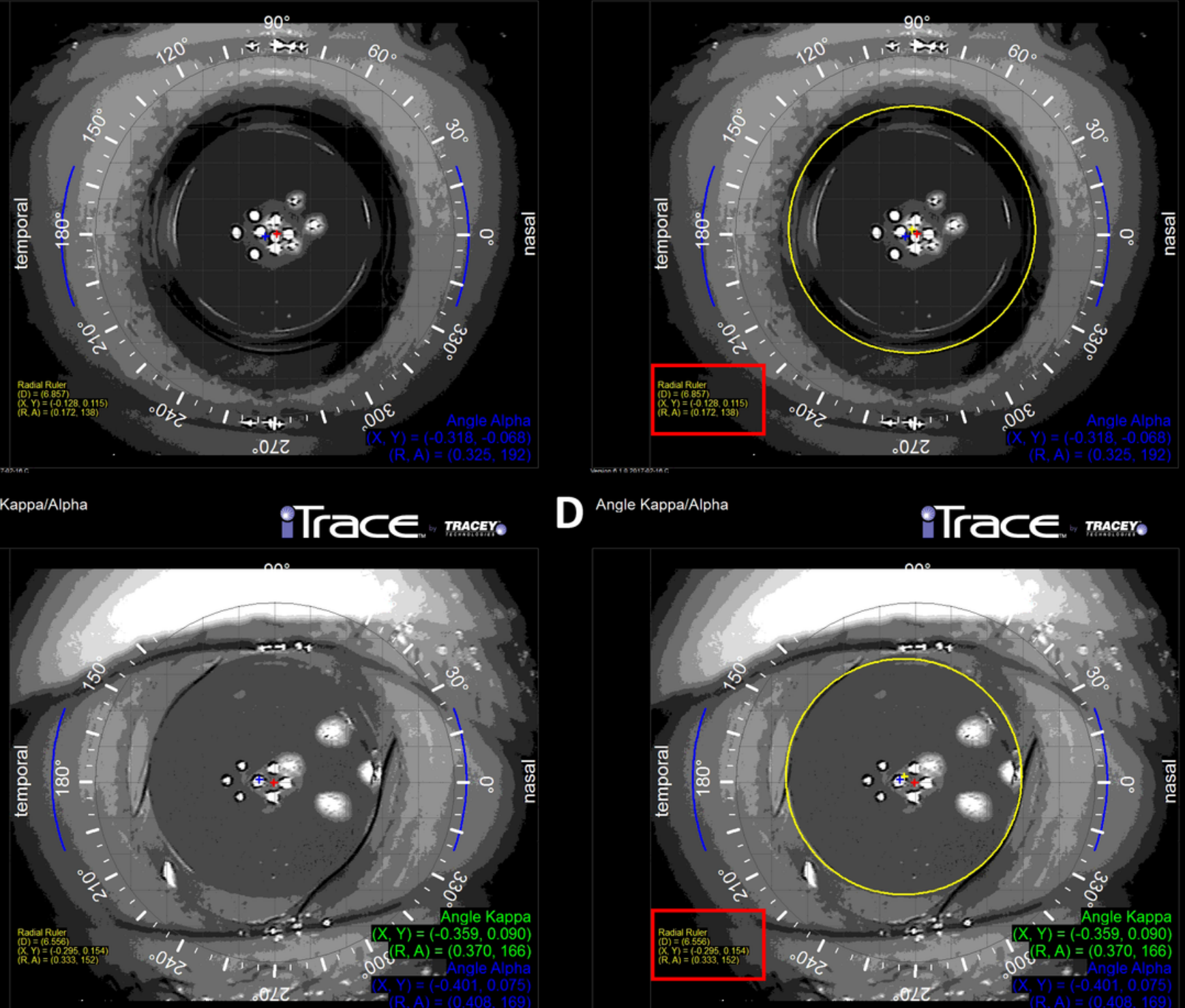

Angle Kappa/Alpha

\section{nemence}

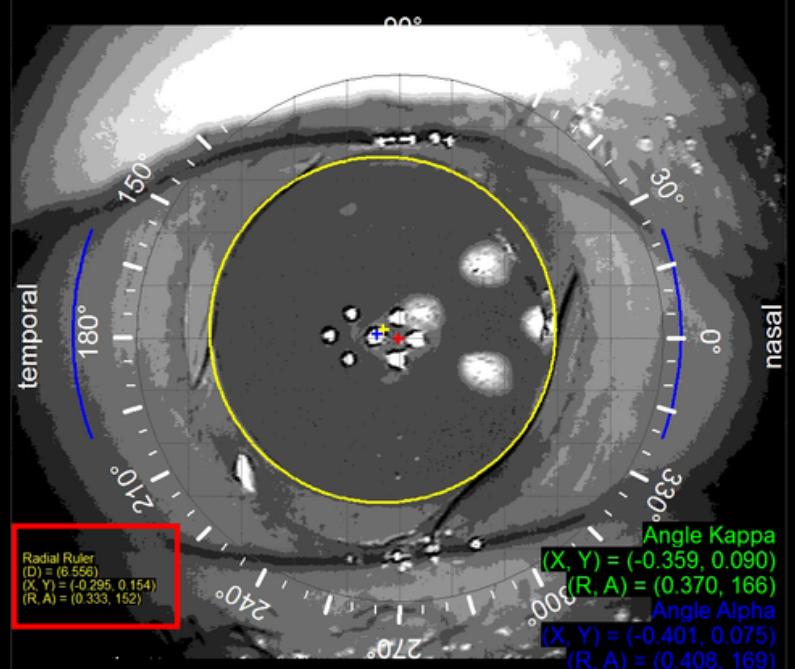

\section{Figure 1}

Center of IOL. The radial ruler tool of iTrace was used to show the edge of the optical zone of the IOL. The distance and orientation of IOL center relative to the visual axis were calculated and shown in polar coordinates. A, B: C-Loop designed IOL, the yellow circle shows edge of IOL optical zone, and the red rectangle shows the magnitude and orientation of CIV. C, D: Plate-haptic designed IOL, the yellow circle shows the edge of IOL optical zone, and the red rectangle shows the magnitude and orientation of CIV. CIV: Center of IOL relative to the visual axis. 
A

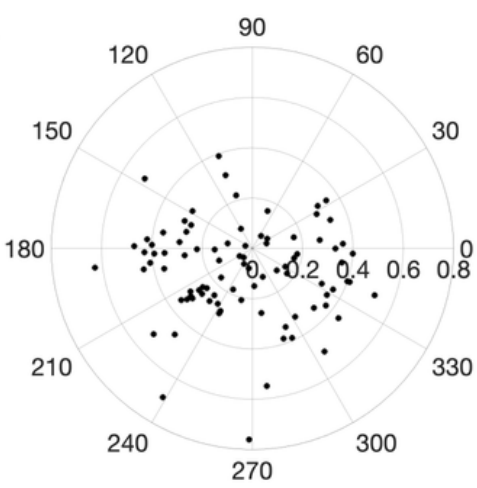

Angle kappa of both eye

D

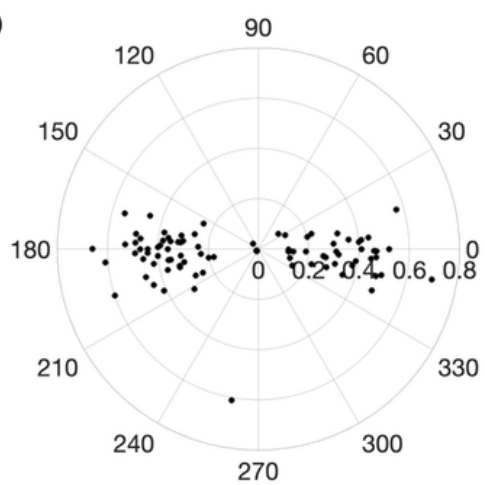

Angle alpha of both eye

G

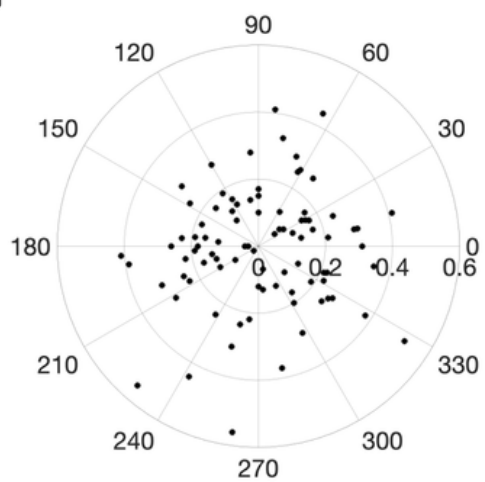

CIV of both eye
B

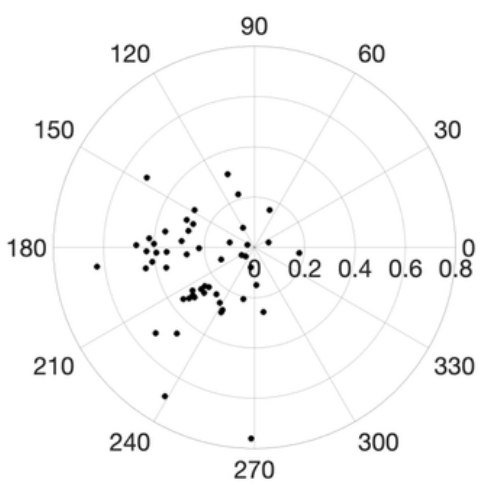

Angle kappa of right eye

E

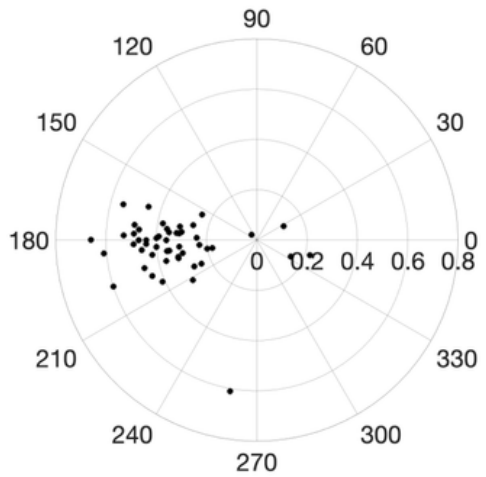

Angle alpha of right eye

H

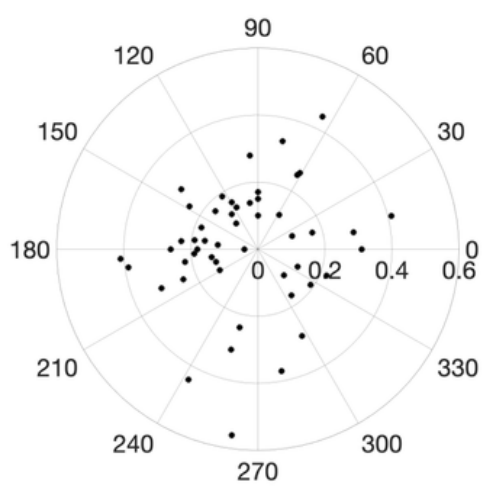

CIV of right eye
C
$120 \quad 90 \quad 60$

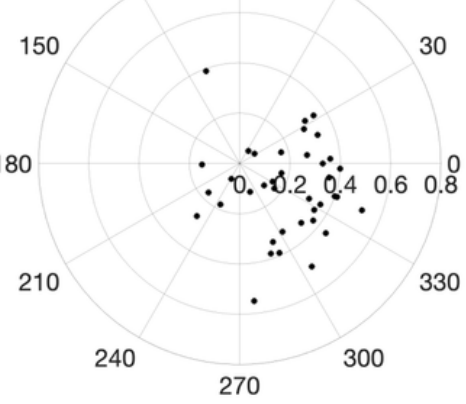

Angle kappa of left eye

$\mathbf{F}$

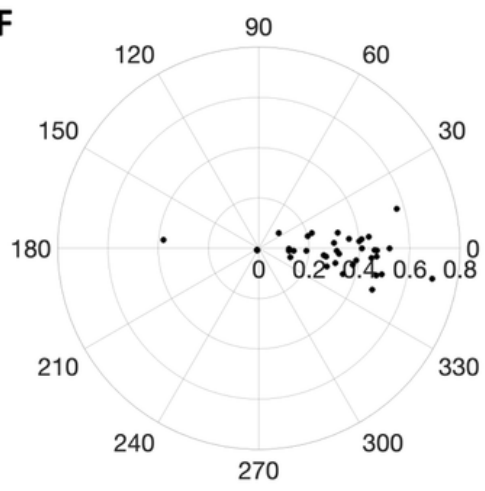

Angle alpha of left eye

I

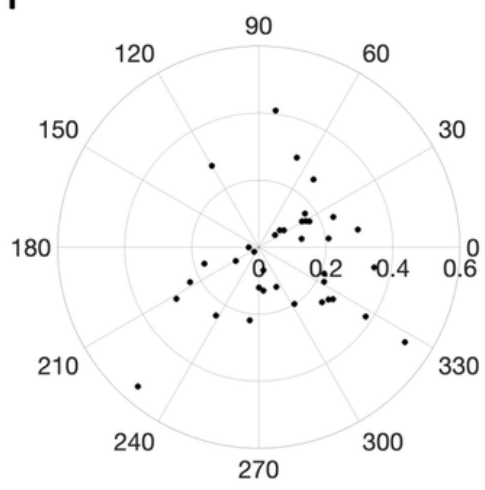

CIV of right eye

\section{Figure 2}

Distribution of angle kappa, angle alpha and CIV. A: Distribution of angle kappa bilaterally. B and C: Distribution of angle kappa unilaterally. Locations of angle kappa were scattered in all directions centered on corneal light reflection. D: Distribution of angle alpha bilaterally. E and F: Distribution of angle alpha unilaterally. Locations of angle alpha were clustered around the horizontal line on both sides of corneal light reflection. G: Distribution of CIV bilaterally. $\mathrm{H}$ and I: Distribution of CIV unilaterally. Locations of CIV show a more diffuse distribution.CIV: the center of IOL relative to the visual axis. $(n=91)$ 
A

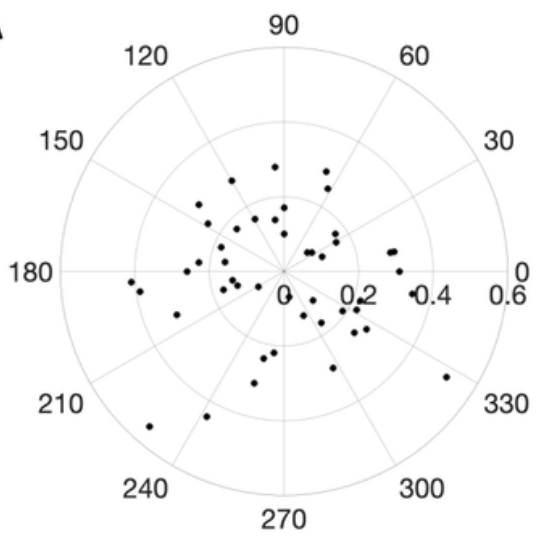

CIV of C-loop IOL in both eye

D

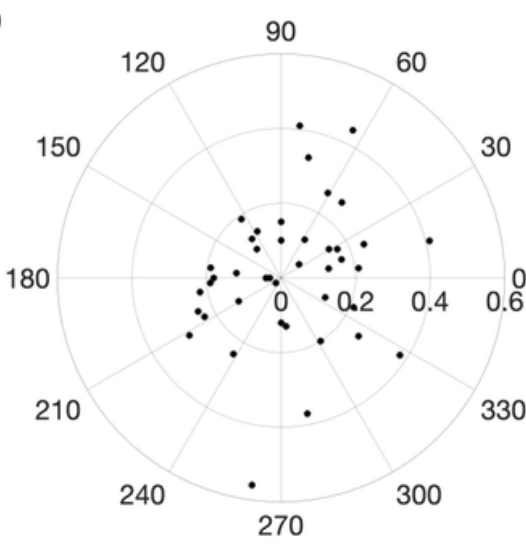

CIV of plate IOL in both eye
B

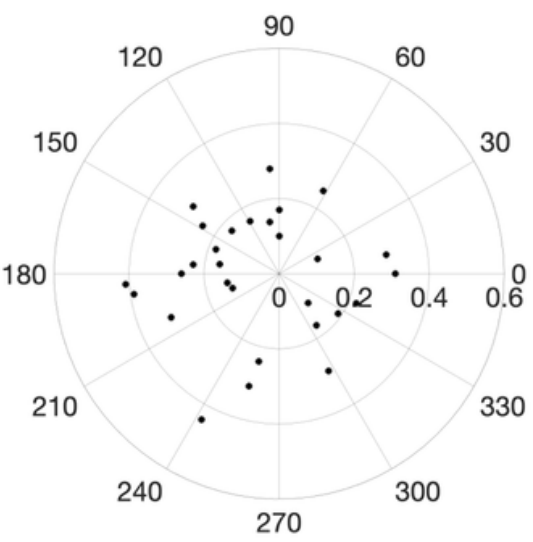

CIV of C-loop IOL in right eye

E

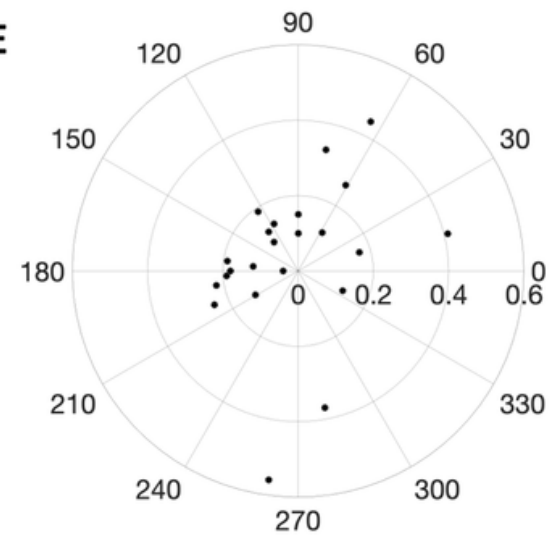

CIV of plate IOL in right eye

0
C

$120 \quad 90 \quad 60$

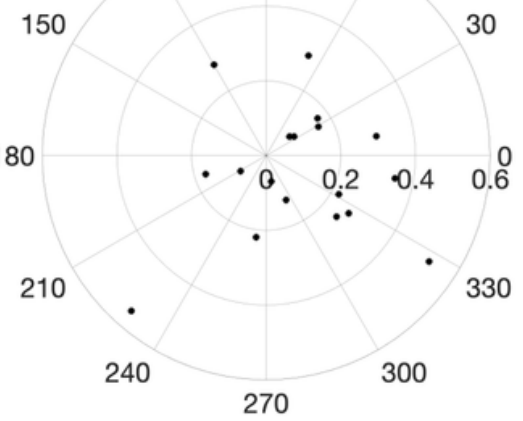

CIV of C-loop IOL in left eye

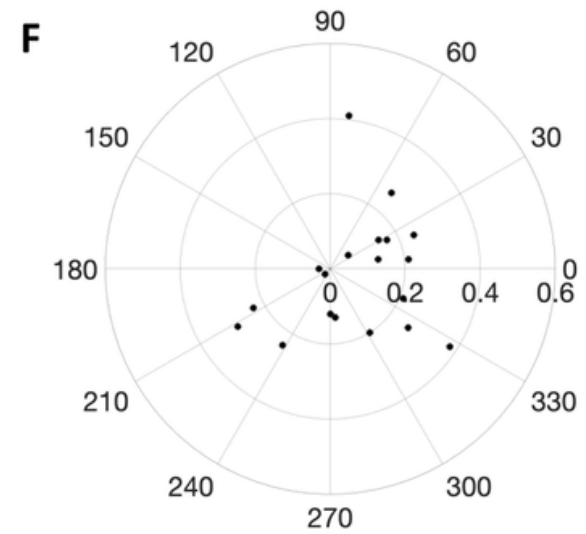

CIV of plate IOL in left eye

\section{Figure 3}

Distribution of CIV of Two Different Designed IOL. A: Distribution of CIV of C-loop design IOL bilaterally. B and C:

Distribution of CIV of C-loop design IOL unilaterally. D: Distribution of CIV of plate-haptic design IOL bilaterally. E and F:

Distribution of CIV of plate-haptic design IOL unilaterally. Locations of CIV were scattered in all directions centered on corneal light reflection bilaterally and unilaterally for both C-loop design IOL and plate-haptic design IOL. CIV: Center of IOL relative to the visual axis. $(n=91)$

\section{Supplementary Files}

This is a list of supplementary files associated with this preprint. Click to download.

- STROBEchecklistcrosssectional1.doc 